\title{
Permanence and extinction in
}

\section{non-autonomous logistic system with random perturbation and feedback control}

Hongxiao $\mathrm{Hu}^{1 *}$ (D) and Ling Zhu

\section{"Correspondence:}

hhxiao1@126.com

${ }^{1}$ College of Science, University of

Shanghai for Science and

Technology, Shanghai, 200093,

P.R. China

Full list of author information is

available at the end of the article

\begin{abstract}
In this paper, we study a stochastic non-autonomous logistic system with feedback control. Sufficient conditions for stochastic asymptotically bounded, extinction, non-persistence in the mean, weak persistence, and persistence in the mean are established. The critical number between weak persistence and extinction is obtained. A very important fact is found in our results, that is, the feedback control is harmless to the permanence of species under the randomized environment.
\end{abstract}

Keywords: non-autonomous logistic model; feedback control; stochastic asymptotically bounded; persistence; extinction

\section{Introduction}

The classical non-autonomous logistic equation can be expressed as follows:

$$
\dot{x}(t)=x(t)(r(t)-a(t) x(t)),
$$

where $x(t)$ denotes the population size at time $t, r(t)$ is the intrinsic growth rate and $r(t) / a(t)$ is the carrying capacity at time $t$. It has been studied extensively and many important results on the global dynamics of solutions have been found (see [1-5] and references therein). On the other hand, sometimes we should search for certain schemes (such as a harvesting procedure or biological control) to ensure the system still have the same dynamic property as system (1.1) under the same conditions. For this reason, many authors considered the controlled system. In [6], Gopalsamy and Weng motivated by control theory and studied the global asymptotic stability of positive equilibrium of a regulated logistic growth with a delay in the state feedback of the control model. In [7], by constructing a suitable Lyapunov functional, the global stability of a single species model with feedback control and distributed time delay were studied. By using coincidence degree theory, some excellent results (see [8-10]) which were concerned with the existence of periodic solution of single species with feedback control are obtained. In many works (see [11-13]), the authors obtained the result that the feedback controls are harmless to the permanence for the deterministic systems.

However, population systems in the real world are often affected by environmental noise. It is important to discover whether the presence of a such noise affects these results (see

(c) $2016 \mathrm{Hu}$ and Zhu. This article is distributed under the terms of the Creative Commons Attribution 4.0 International License (http://creativecommons.org/licenses/by/4.0/), which permits unrestricted use, distribution, and reproduction in any medium, provided you give appropriate credit to the original author(s) and the source, provide a link to the Creative Commons license, and indicate if changes were made. 
[14-16]). Recently many authors have discussed population systems subject to white noise (see [14-22]). Recall that $r(t)$ represents the intrinsic growth rate at time $t$. In practice we usually estimate it by an average value plus an error term. In general, by the well-known central limit theorem, the error term follows a normal distribution. Thus, for a short correlation time, we may replace $r(t)$ by

$$
r(t) \rightarrow r(t)+\sigma(t) \dot{B}(t)
$$

where $\dot{B}(t)$ is white noise and $\sigma(t)$ is a positive number representing the intensity of the noise at time $t$. Then (1.1) becomes a stochastic differential equation

$$
\mathrm{d} x(t)=x(t)(r(t)-a(t) x(t)) \mathrm{d} t+\sigma(t) x(t) \mathrm{d} B(t)
$$

In [23], the authors considered the case that the coefficients of (1.2) are all periodic functions with period $T$. They obtained the stochastic permanence of (1.2) and global attractivity of one positive solution $x^{p}(t)$ satisfying $\mathrm{E}\left[1 / x^{p}(t)\right]=\mathrm{E}\left[1 / x^{p}(t+T)\right]$. In [22], Liu and Wang improved the permanence results in [23], and obtained the critical number between weak persistence and extinction. However, to the best of the authors' knowledge, to this day, still few scholars consider the stochastic perturbation logistic system with feedback controls. In fact, we have known very little about how feedback controls affect the survival of species which is under the randomized environment.

So, motivated by the above analysis, we will study the following non-autonomous randomized logistic system with feedback control:

$$
\left\{\begin{array}{l}
\mathrm{d} x(t)=x(t)(r(t)-a(t) x(t)-c(t) u(t)) \mathrm{d} t+\sigma(t) x(t) \mathrm{d} B_{t}, \\
\mathrm{~d} u(t)=(-e(t) u(t)+f(t) x(t)) \mathrm{d} t
\end{array}\right.
$$

where $r(t)$ is a continuous bounded function on $[0,+\infty)$ and $a(t), c(t), \sigma(t), e(t)$, and $f(t)$ are nonnegative continuous bounded function on $[0,+\infty)$. Throughout this paper, for system (1.3) we introduce the following hypotheses:

$\left(\mathrm{H}_{1}\right)$ There is a positive constant $\lambda$ such that

$$
\liminf _{t \rightarrow \infty} \int_{t}^{t+\lambda} a(s) \mathrm{d} s>0 .
$$

$\left(\mathrm{H}_{2}\right)$ There is a positive constant $\gamma_{1}$ such that

$$
\liminf _{t \rightarrow \infty} \int_{t}^{t+\gamma_{1}} e(s) \mathrm{d} s>0 .
$$

$\left(\mathrm{H}_{3}\right)$ There is a positive constant $\gamma_{2}$ such that

$$
\liminf _{t \rightarrow \infty} \int_{t}^{t+\gamma_{2}} f(s) \mathrm{d} s>0
$$

In this work, our purpose is to establish the sufficient conditions for asymptotically bounded, extinction, non-persistence in the mean, weak persistence and persistence in the mean of system (1.3). We will find that, in our results, the feedback control is harmless to the permanence of species with stochastic perturbation. 


\section{Preliminaries}

Throughout this paper, unless otherwise specified, let $\left(\Omega, \mathcal{F},\left\{\mathcal{F}_{t}\right\}_{t \geq 0}, \mathbb{P}\right)$ be a complete probability space with a filtration $\left\{\mathcal{F}_{t}\right\}_{t \geq 0}$ satisfying the usual conditions (i.e. it is right continuous and $\mathcal{F}_{0}$ contains all P-null set). Let $B(t), t \geq 0$, be 1 -dimension standard Brownian motion defined on this probability space. We also denote by $R_{+}$the interval $[0,+\infty)$, and denote by $R_{+}^{2}$ the set $\{(x, y) \mid x>0, y>0\}$. For convenience and simplicity in the following discussion, define

$$
\begin{array}{ll}
f_{u}=\sup _{s \in R_{+}} f(s), \quad f_{l}=\inf _{s \in R_{+}} f(s), & \langle f(t)\rangle=\frac{1}{t} \int_{0}^{t} f(s) \mathrm{d} s, \\
\langle f\rangle^{*}=\limsup _{t \rightarrow \infty} \frac{1}{t} \int_{0}^{t} f(s) \mathrm{d} s \text { and } \quad\langle f\rangle_{*}=\liminf _{t \rightarrow \infty} \frac{1}{t} \int_{0}^{t} f(s) \mathrm{d} s,
\end{array}
$$

where $f(s)$ is a continuous bounded function on $R_{+}$.

Now, we introduce several lemmas which will be very useful in the proofs of the main results. We consider the following randomized non-autonomous logistic equation:

$$
\mathrm{d} N(t)=N(t)((m(t)-n(t) N(t)) \mathrm{d} t+\alpha(t) \mathrm{d} B(t))
$$

We have the following results which can be found in [24].

Lemma 2.1 Suppose $m(t), n(t)$, and $\alpha(t)$ are continuous bounded functions on $R_{+}$and $n(t)$ is nonnegative on $R_{+}$. Then there exists a unique continuous positive solution $N(t)$ to system (2.1) for any positive initial value $N(0)=N_{0}$, which is global and represented by

$$
N(t)=\frac{\exp \left\{\int_{0}^{t}\left(m(s)-\frac{\alpha^{2}(s)}{2}\right) \mathrm{d} s+\int_{0}^{t} \alpha(s) \mathrm{d} B(s)\right\}}{1 / N_{0}+\int_{0}^{t} n(s) \exp \left\{\int_{0}^{s}\left(m(\tau)-\frac{\alpha^{2}(\tau)}{2}\right) \mathrm{d} \tau+\int_{0}^{s} \alpha(\tau) \mathrm{d} B(\tau)\right\} \mathrm{d} s} .
$$

Remark 2.1 In [24], the authors obtained the same results as Lemma 2.1 with conditions $m(t), n(t), \alpha(t)>0$. But checking the proof in Theorem 2.2 in [24], we can obtain the same results in Lemma 2.1, only $n(t)$ needs to be nonnegative.

We consider the following non-autonomous differential equation:

$$
\frac{\mathrm{d} y(t)}{\mathrm{d} t}=y^{\alpha}(t)\left(m(t)-n(t) y^{\beta}(t)\right),
$$

where $m(t)$ and $n(t)$ are continuous bounded function on $R_{+}$. We have the following results for system (2.2).

Lemma 2.2 Suppose that there are positive constants $\theta$ and $\gamma$ such that

$$
\liminf _{t \rightarrow \infty} \int_{t}^{t+\theta} m(s) \mathrm{d} s>0 \text { and } \liminf _{t \rightarrow \infty} \int_{t}^{t+\gamma} n(s) \mathrm{d} s>0 .
$$

Assume $\beta>0$ and one of the following conditions is satisfied:

(a) $\alpha=1$ and $n(t)$ is nonnegative;

(b) $\alpha+\beta=1, \alpha \geq 0$, and $m(t)$ is nonnegative. 


\section{Then we have}

(i) for any given initial value $y_{0}>0$, there is a unique solution $y(t)$ of (2.2) which is global positive;

(ii) there exist positive constants $l$ and $L$ such that

$$
l \leq \liminf _{t \rightarrow \infty} y(t) \leq \limsup _{t \rightarrow \infty} y(t) \leq L
$$

for any positive solution $y(t)$ of equation (2.2);

(iii) for any two positive solutions $x(t)$ and $y(t)$ of system (2.2) we have

$$
\lim _{t \rightarrow \infty}(x(t)-y(t))=0 .
$$

Proof If $\alpha=1$, it is obviously that system (2.2) has a unique global positive solution for any positive initial value. And we can prove the conclusion (ii) of this lemma similar to Lemma 1 in [25]. Now, we prove the conclusion (iii) for this case. Let $x(t)$ and $y(t)$ be any two solutions of equation (2.2). By conclusion (ii), there are positive constants $l$ and $L$ such that $l \leq x(t), y(t) \leq L$ for all $t \geq t_{0}$. We can choose the Lyapunov function $V(t)=$ $|\ln x(t)-\ln y(t)|$. By calculating the upper derivative of $V(t)$ and using the mean value theorem of differential, we have

$$
\begin{aligned}
D^{+} V(t) & \leq-n(t)\left|x^{\beta}(t)-y^{\beta}(t)\right| \\
& =-\beta n(t) \xi^{\beta-1}(t)|x(t)-y(t)| \\
& \leq-q n(t) V(t) \quad \text { for all } t \geq t_{0},
\end{aligned}
$$

where $\xi(t)$ is between $x(t)$ and $y(t)$, and

$$
q= \begin{cases}\beta l^{\beta}, & \text { if } \beta \geq 1, \\ \beta l L^{\beta-1}, & \text { if } \beta<1 .\end{cases}
$$

Since $\int_{0}^{\infty} n(s) \mathrm{d} s=+\infty$, we have $V(t) \rightarrow 0$ as $t \rightarrow \infty$. Therefore,

$$
\lim _{t \rightarrow \infty}(x(t)-y(t))=0 .
$$

This completes the proof of the case (a).

Now, we prove the case (b). From system (2.2) we have

$$
\frac{\mathrm{d}}{\mathrm{d} t} y^{\beta}(t)=\beta\left(m(t)-n(t) y^{\beta}(t)\right) .
$$

We denote $z(t)=y^{\beta}(t)$, and this yields

$$
\frac{\mathrm{d} z(t)}{\mathrm{d} t}=\beta(m(t)-n(t) z(t)) .
$$

Let $w(t)=1 / z(t)$, we obtain

$$
\frac{\mathrm{d} w(t)}{\mathrm{d} t}=\beta w(t)(n(t)-m(t) w(t)) .
$$


Consequently, (i) of this lemma holds. By Lemma 1 of [25], we can find that system (2.4) has the following results:

(i) there exist positive constants $l$ and $L$ such that

$$
l \leq \liminf _{t \rightarrow \infty} w(t) \leq \limsup _{t \rightarrow \infty} w(t) \leq L
$$

for any positive solution $w(t)$ of equation (2.4);

(ii) for any two positive solutions $w_{1}(t)$ and $w_{2}(t)$ of system (2.4) we have

$$
\lim _{t \rightarrow \infty}\left(w_{1}(t)-w_{2}(t)\right)=0 .
$$

Therefore, the conclusions (ii) and (iii) of this lemma hold if (b) arises. This completes the proof of the lemma.

Remark 2.2 In [25], the authors considered the case $\alpha=\beta=1$ of system (2.2), and obtained the same conclusions with this lemma. Hence, their results are generalized by Lemma 2.2.

Remark 2.3 If $m_{l}$ and $n_{l}$ are positive, it is easy to find that

$$
\frac{m_{l}}{n_{u}} \leq \liminf _{t \rightarrow \infty} y^{\beta}(t) \leq \limsup _{t \rightarrow \infty} y^{\beta}(t) \leq \frac{m_{u}}{n_{l}}
$$

for any positive solution $y(t)$ of equation (2.2).

Now, we consider the following non-autonomous linear equation:

$$
\frac{\mathrm{d} y(t)}{\mathrm{d} t}=m(t)-n(t) y(t)+p(t)
$$

where functions $m(t), n(t)$, and $p(t)$ are bounded continuous defined on $R_{+}$and $m(t)$ and $n(t)$ are nonnegative for all $t \geq 0$. Suppose that $v(t)$ is the solution of the following equation:

$$
\frac{\mathrm{d} v(t)}{\mathrm{d} t}=m(t)-n(t) v(t)
$$

with initial condition $v(0)=1$. We have the following useful result which can be found in [26].

Lemma 2.3 Suppose that there exists a constant $\omega>0$ such that

$$
\liminf _{t \rightarrow \infty} \int_{t}^{t+\omega} n(s) \mathrm{d} s>0 .
$$

Then, for any constants $\varepsilon>0$ and $M>0$ there exist constants $\delta=\delta(\varepsilon)>0$ and $T_{0}=T_{0}(M)>$ 0 such that for any $t_{0} \in R_{+}, v_{0} \in R$, and $\left|y_{0}\right| \leq M$, when $|p(t)|<\delta$ for all $t \geq t_{0}$, one has

$$
\left|y\left(t, t_{0}, y_{0}\right)-v(t)\right|<\varepsilon \quad \text { for all } t \geq t_{0}+T_{0}
$$

where $y\left(t, t_{0}, y_{0}\right)$ is the solution of equation (2.5) with initial condition $y\left(t_{0}\right)=y_{0}$. 
Further, we consider the following non-autonomous equation:

$$
\frac{\mathrm{d} y(t)}{\mathrm{d} t}=y^{\alpha}(t)\left(m(t)-n(t) y^{\beta}(t)+p(t)\right)
$$

where $\alpha \geq 0, \beta>0, \alpha+\beta=1$, the functions $m(t), n(t)$, and $p(t)$ are bounded continuous defined on $R_{+}$and $m(t)$ and $n(t)$ are nonnegative for all $t \geq 0$. Suppose that $v(t)$ is the solution of the following equation:

$$
\frac{\mathrm{d} v(t)}{\mathrm{d} t}=v^{\alpha}(t)\left(m(t)-n(t) v^{\beta}(t)\right)
$$

with initial condition $v(0)=1$. We have the following result.

Lemma 2.4 Suppose that there exists a constant $\gamma>0$ such that

$$
\liminf _{t \rightarrow \infty} \int_{t}^{t+\gamma} n(s) \mathrm{d} s>0
$$

Then, for any constants $\varepsilon>0$ and $M>0$ there exist constants $\delta=\delta(\varepsilon)>0$ and $T_{0}=T_{0}(M)>$ 0 such that for any $t_{0} \in R_{+}$and $0<y_{0}<M$, when $|p(t)|<\delta$ for all $t \geq t_{0}$, one has

$$
\left|y^{\beta}\left(t, t_{0}, y_{0}\right)-v^{\beta}(t)\right|<\varepsilon \quad \text { for all } t \geq t_{0}+T_{0},
$$

where $y\left(t, t_{0}, y_{0}\right)$ is the solution of system (2.6) with initial condition $y\left(t_{0}\right)=y_{0}$.

Proof If $\alpha=0$, we have $\beta=1$. This case is the same as Lemma 2.3. If $\alpha \neq 0$, we let $\tilde{y}(t)=y^{\beta}(t)$ and $\tilde{v}(t)=v^{\beta}(t)$, from (2.6) and (2.7) we have

$$
\frac{\mathrm{d} \tilde{y}(t)}{\mathrm{d} t}=\beta(m(t)-n(t) \tilde{y}(t)+p(t))
$$

and

$$
\frac{\mathrm{d} \tilde{v}(t)}{\mathrm{d} t}=\beta(m(t)-n(t) \tilde{v}(t)) .
$$

Then, using Lemma 2.3, we can obtain the conclusion of this lemma.

Remark 2.4 In Lemma 2.3, the authors discussed the case $\alpha=0$ and $\beta=1$ of this lemma. Hence, their results are extended by this lemma.

\section{Asymptotically bounded of the global positive solution}

In system (1.3), $x(t)$ is the size of the species and $u(t)$ is the regulator, thus we are only interested in the positive solutions. Moreover, in order for a stochastic differential equation to have a unique global (i.e. no explosion in a finite time) solution for any given initial value, the coefficients of the equation are generally required to satisfy the linear growth condition and local Lipschitz condition ( $c f$. Mao [27]). However, the coefficients of system (1.3) do not satisfy the linear growth condition, though they are locally Lipschitz continuous. In this section, using the comparison theorem of stochastic equations (see [28]) we will show there is a unique positive solution with positive initial value of system (1.3). 
Theorem 3.1 For any given initial value $\left(x_{0}, u_{0}\right) \in R_{+}^{2}$, there is a unique solution $(x(t), u(t))$ to system (1.3) on $t \geq 0$ and the solution will remain in $R_{+}^{2}$ with probability one, namely $(x(t), u(t)) \in R_{+}^{2}$ for all $t \geq 0$ almost surely.

Proof Since the coefficients of the equation are locally Lipschtiz continuous, it is known that for any given initial value $\left(x_{0}, u_{0}\right) \in R_{+}^{2}$ there is a unique maximal local solution $(x(t), u(t))$ for all $t \in\left[0, \tau_{e}\right)$ where $\tau_{e}$ is the explosion time. Furthermore, by Lemma 2.1, we have

$$
x(t)=\frac{\exp \left\{\int_{0}^{t}[b(s)-c(s) u(s)] \mathrm{d} s+\int_{0}^{t} \sigma(s) \mathrm{d} B(s)\right\}}{1 / x_{0}+\int_{0}^{t} a(s) \exp \left\{\int_{0}^{s}[b(\tau)-c(s) u(s)] \mathrm{d} \tau+\int_{0}^{s} \sigma(\tau) \mathrm{d} B(\tau)\right\} \mathrm{d} s}>0
$$

and

$$
u(t) \geq u_{0} \exp \left\{-\int_{0}^{t} e(s) \mathrm{d} s\right\}>0 \quad \text { for all } t \in\left[0, \tau_{e}\right),
$$

where $b(t)=r(t)-0.5 \sigma^{2}(t)$. Hence, to show this solution is globally positive, we only to show that $\tau_{e}=\infty$ a.s. By the first equation of (1.3) we have

$$
\mathrm{d} x(t) \leq x(t)(r(t)-a(t) x(t)) \mathrm{d} t+\sigma(t) x(t) \mathrm{d} B(t)
$$

Consider the following auxiliary equation:

$$
\left\{\begin{array}{l}
\mathrm{d} y(t)=y(t)(r(t)-a(t) y(t)) \mathrm{d} t+\sigma(t) y(t) \mathrm{d} B(t) \\
y(0)=x_{0}
\end{array}\right.
$$

From Lemma 2.1, we know that there exists a unique continuous positive solution $y(t)$ of system (3.2) for any positive initial value $x_{0}$, which will remain in $R_{+}$with probability one. Consequently, by the comparison theorem of stochastic differential equation we have

$$
x(t) \leq y(t) \text { for all } t \geq 0 \text { a.s. }
$$

Therefore, $x(t)<\infty$ for all $t>0$ a.s. By the second equation of (1.3) we can represent $u(t)$ by

$$
u(t)=\left(\int_{0}^{t} f(s) x(s) \exp \left\{\int_{0}^{s} e(\tau) \mathrm{d} \tau\right\}+u_{0}\right) \exp \left\{-\int_{0}^{t} e(s) \mathrm{d} s\right\} .
$$

From this we can find that if $x(t)$ is global, then $u(t)$ also is a global solution, i.e. $\tau_{e}=\infty$ a.s. This complete the proof of the theorem.

Now, we will discuss the asymptotically bounded property of the unique global positive solution of system (1.3). To be precise, let us now give the definition of asymptotically bounded.

Definition 3.1 Let $p>0$, system (1.3) is said to be asymptotically bounded in $p$ th moment if there are positive constants $H=H(p)$ and $K=K(p)$ such that

$$
\limsup _{t \rightarrow \infty} \mathrm{E}\left[\left|x\left(t ; t_{0}, x_{0}, u_{0}\right)\right|^{p}\right] \leq H \quad \text { and } \quad \limsup _{t \rightarrow \infty} \mathrm{E}\left[\left|u\left(t ; t_{0}, x_{0}, u_{0}\right)\right|^{p}\right] \leq K
$$

for all $\left(x_{0}, u_{0}\right) \in R_{+}^{2}$. 
Theorem 3.2 Suppose $\left(\mathrm{H}_{1}\right)-\left(\mathrm{H}_{3}\right)$ hold, for any $p \geq 1$ there is a positive constant $\mu$ such that

$$
\liminf _{t \rightarrow \infty} \int_{t}^{t+\mu}\left(r(s)+\frac{1}{2}(p-1) \sigma^{2}(s)\right) \mathrm{d} s>0 .
$$

Then system (1.3) is asymptotically bounded in pth moment. Furthermore, we have

$$
\limsup _{t \rightarrow \infty} \mathrm{E}\left[x^{p}(t)\right] \leq \limsup _{t \rightarrow \infty} y^{*}(t) \quad \text { and } \quad \limsup _{t \rightarrow \infty} \mathrm{E}\left[u^{p}(t)\right] \leq \limsup _{t \rightarrow \infty} v^{*}(t)
$$

where $y^{*}(t)$ is the solution of the equation

$$
\frac{\mathrm{d} y(t)}{\mathrm{d} t}=p y(t)\left(\left(r(t)+\frac{1}{2}(p-1) \sigma^{2}(t)\right)-a(t) y^{\frac{1}{p}}(t)\right)
$$

with initial value $y^{*}(0)=1$, and $v^{*}(t)$ is the solution of the equation

$$
\frac{\mathrm{d} v(t)}{\mathrm{d} t}=p v^{\frac{p-1}{p}}(t)\left(-e(t) v^{\frac{1}{p}}(t)+f(t) y^{* \frac{1}{p}}(t)\right)
$$

with initial value $v^{*}(0)=1$.

Proof Applying Itô's formula to $x^{p}(t)$, we have

$$
\mathrm{d} x^{p}(t)=p x^{p}(t)\left(\left(r(t)+\frac{1}{2}(p-1) \sigma^{2}(t)-a(t) x(t)-c(t) u(t)\right) \mathrm{d} t+\sigma(t) \mathrm{d} B(t)\right) .
$$

For every integer $n \geq 1$, define the stopping time

$$
\tau_{n}=\inf \{t \geq 0:|x(t)| \geq n\}
$$

Clearly, $\tau_{n} \uparrow \infty$ a.s. Integrating from 0 to $t \wedge \tau_{n}$ and taking expectations yield

$$
\mathrm{E}\left[x^{p}\left(t \wedge \tau_{n}\right)\right]-\mathrm{E}\left[x_{0}^{p}\right]=p \int_{0}^{t \wedge \tau_{n}} \mathrm{E}\left[x^{p}(s)\left(\left(r(s)+\frac{1}{2}(p-1) \sigma^{2}(s)-a(s) x(s)-c(s) u(s)\right)\right)\right] \mathrm{d} s .
$$

Letting $n \rightarrow \infty$, and by the well-known Hölder inequality,

$$
\begin{aligned}
\frac{\mathrm{dE}\left[x^{p}(t)\right]}{\mathrm{d} t} & =p\left(\left(r(t)+\frac{1}{2}(p-1) \sigma^{2}(t)\right) \mathrm{E}\left[x^{p}(t)\right]-a(t) \mathrm{E}\left[x^{p+1}(t)\right]-c(t) \mathrm{E}\left[u(t) x^{p}(t)\right]\right) \\
& \leq p \mathrm{E}\left[x^{p}(t)\right]\left(r(t)+\frac{1}{2}(p-1) \sigma^{2}(t)-a(t)\left(\mathrm{E}\left[x^{p}(t)\right]\right)^{\frac{1}{p}}\right) .
\end{aligned}
$$

By the assumption $\left(\mathrm{H}_{1}\right)$ and (3.3), considering the auxiliary equation (3.4) and using the standard comparison theorem and (a) of Lemma 2.2, we can obtain

$$
\limsup _{t \rightarrow \infty} \mathrm{E}\left[x^{p}(t)\right] \leq \limsup _{t \rightarrow \infty} y^{*}(t):=H(p) .
$$

Furthermore, for any $\alpha_{0}>0$ there exists a constant $T_{1}>0$ such that

$$
\mathrm{E}\left[x^{p}(t)\right] \leq y^{*}(t)+\alpha_{0} \quad \text { for all } t \geq t_{0}+T_{1}
$$


By the second equation of system (1.3) we have

$$
\frac{\mathrm{d} u^{p}(t)}{\mathrm{d} t}=p\left[-e(t) u^{p}(t)+f(t) x(t) u^{p-1}(t)\right]
$$

Integrating from 0 to $t$ and taking expectations, we have

$$
\mathrm{E}\left[u^{p}(t)\right]-\mathrm{E}\left[u_{0}^{p}\right]=p \int_{0}^{t}\left(-e(s) \mathrm{E}\left[u^{p}(s)\right]+f(s) \mathrm{E}\left[x(s) u^{p-1}(s)\right]\right) \mathrm{d} s
$$

So,

$$
\begin{aligned}
\frac{\mathrm{dE}\left[u^{p}(t)\right]}{\mathrm{d} t} & =p\left(-e(t) \mathrm{E}\left[u^{p}(t)\right]+f(t) \mathrm{E}\left[x(t) u^{p-1}(t)\right]\right) \\
& \leq p\left(\mathrm{E}\left[u^{p}(t)\right]\right)^{\frac{p-1}{p}}\left[-e(t)\left(\mathrm{E}\left[u^{p}(t)\right]\right)^{\frac{1}{p}}+f(t)\left(\mathrm{E}\left[x^{p}(t)\right]\right)^{\frac{1}{p}}\right] \\
& \leq p\left(\mathrm{E}\left[u^{p}(t)\right]\right)^{\frac{p-1}{p}}\left[-e(t)\left(\mathrm{E}\left[u^{p}(t)\right]\right)^{\frac{1}{p}}+f(t)\left(y^{*}(t)+\alpha_{0}\right)^{\frac{1}{p}}\right]
\end{aligned}
$$

for all $t \geq t_{0}+T_{1}$. Consider the following comparison equation:

$$
\frac{\mathrm{d} z(t)}{\mathrm{d} t}=p z^{\frac{p-1}{p}}(t)\left[-e(t) z^{\frac{1}{p}}(t)+f(t)\left(y^{*}(t)+\alpha_{0}\right)^{\frac{1}{p}}\right]
$$

By the assumptions $\left(\mathrm{H}_{2}\right)$ and $\left(\mathrm{H}_{3}\right)$ and $(\mathrm{b})$ of Lemma 2.2 we can find that for the solution $z(t)$ of equation (3.6) with initial value $z\left(t_{0}+T_{1}\right)=\mathrm{E}\left[u^{p}\left(t_{0}+T_{1}\right)\right]$ is bounded. Hence, we can denote $M=\sup _{t \in R_{+}} z(t)$. By Lemma 2.4, for any $\varepsilon>0$ and $M$ there exist positive constants $\delta_{0}=\delta_{0}(\varepsilon)$ and $T_{2}=T_{2}(M) \geq T_{1}$ such that for any $t_{0} \in R_{+}$, when $\left|f(t)\left(y^{*}(t)+\alpha_{0}\right)^{\frac{1}{p}}-f(t) y^{* \frac{1}{p}}(t)\right|<\delta_{0}$ for all $t \geq t_{0}$, we have

$$
\left|z^{\frac{1}{p}}(t)-v^{* \frac{1}{p}}(t)\right| \leq \varepsilon
$$

By the comparison theorem of differential equation, we can obtain from (3.5) and (3.7)

$$
\mathrm{E}\left[u^{p}(t)\right] \leq\left(v^{* \frac{1}{p}}(t)+\varepsilon\right)^{p}
$$

for all $t \geq t_{0}+T_{2}$. Since $\varepsilon$ is arbitrary, we can obtain

$$
\limsup _{t \rightarrow \infty} \mathrm{E}\left[u^{p}(t)\right] \leq \limsup _{t \rightarrow \infty} v^{*}(t):=K(p)
$$

This completes the proof of the theorem.

In the following, we denote $q(t)=r(t)+0.5(p-1) \sigma^{2}(t)$.

Remark 3.1 If $q_{u}, a_{l}$, and $e_{l}$ are positive, we can choose

$$
H(p)=\frac{q_{u}^{p}}{a_{l}^{p}} \quad \text { and } \quad K(p)=\frac{f_{u}^{p} H(p)}{e_{l}^{p}}
$$

which will be discussed in the following corollary. 
Corollary 3.1 Suppose $q_{u}, a_{l}$, and $e_{l}$ are positive. Then system (1.3) is asymptotically bounded in the pth moment for any $p \geq 1$. Furthermore,

$$
\limsup _{t \rightarrow \infty} \mathrm{E}\left[x^{p}(t)\right] \leq \frac{q_{u}^{p}}{a_{l}^{p}} \text { and } \quad \limsup _{t \rightarrow \infty} \mathrm{E}\left[u^{p}(t)\right] \leq \frac{f_{u}^{p} q_{u}^{p}}{a_{l}^{p} e_{l}^{p}}
$$

Remark 3.2 If $c(t) \equiv 0$, we can obtain a randomized logistic equation without feedback control

$$
\mathrm{d} x(t)=x(t)(r(t)-a(t) x(t)) \mathrm{d} t+\sigma(t) x(t) \mathrm{d} B(t) .
$$

From Theorem 3.2, if $\left(\mathrm{H}_{1}\right)$ and (3.3) hold, then system (3.8) is asymptotically bounded in $p$ th moment. In [23], the authors studied the stochastic bounded of system (3.8) with the assumptions $r_{l}>0$ and $a_{l}>0$. Hence, our conditions in Theorem 3.2 are weaker than that in [23].

Definition 3.2 System (1.3) is said to be stochastically ultimately bounded, if for any $\varepsilon \in$ $(0,1)$ there is a positive constant $\chi(=\chi(\varepsilon))$ such that the solution of SDE $(1.3)$ with any positive initial value has the property that

$$
\limsup _{t \rightarrow \infty} \mathbb{P}(x(t)>\chi)<\varepsilon \text { and } \quad \limsup _{t \rightarrow \infty} \mathbb{P}(u(t)>\chi)<\varepsilon .
$$

Theorem 3.3 Suppose assumptions $\left(\mathrm{H}_{1}\right)-\left(\mathrm{H}_{3}\right)$ hold, and for some $p \geq 1$ and $\mu>0$ such that

$$
\liminf _{t \rightarrow \infty} \int_{t}^{t+\mu}\left(r(s)+\frac{1}{2}(p-1) \sigma^{2}(s)\right) \mathrm{d} s>0 .
$$

Then system (1.3) is stochastically ultimately bounded.

Proof This can easily be verified by Chebyshev's inequality and Theorem 3.2.

Corollary 3.2 Suppose $a_{l}$ and $e_{l}$ are positive, and for some $p \geq 1$ such that $q_{u}>0$. Then solution of system (1.3) are stochastically ultimately bounded.

Remark 3.3 From Theorems 3.2 and 3.3, we can find that the asymptotically bounded property of system (1.3) cannot be changed by the feedback control even though the system is randomized by the environment.

\section{Extinction and persistence in time average}

Now, we will discuss extinction and persistence of system (1.3). For any positive solution $(x(t), u(t))$ of system (1.3) we first introduce some useful definitions.

Definition 4.1 System (1.3) is said to be extinction almost surely, if

$$
\lim _{t \rightarrow \infty} x(t)=0 \quad \text { and } \quad \lim _{t \rightarrow \infty} u(t)=0 \quad \text { a.s.; }
$$


non-persistence in the mean, if

$$
\lim _{t \rightarrow \infty}\langle x(t)\rangle=0 \quad \text { and } \quad \lim _{t \rightarrow \infty}\langle u(t)\rangle=0 \quad \text { a.s.; }
$$

uniform persistence in the mean, if there are positive constants $m$ and $M$ such that

$$
m \leq\langle x\rangle_{*} \leq\langle x\rangle^{*} \leq M \quad \text { and } \quad m \leq\langle u\rangle_{*} \leq\langle u\rangle^{*} \leq M \quad \text { a.s. }
$$

For convenience and simplicity in the following discussion, we denote $b(t)=r(t)-$ $0.5 \sigma^{2}(t)$ and $(x(t), u(t))=\left(x\left(t, 0, x_{0}, u_{0}\right), u\left(t, 0, x_{0}, u_{0}\right)\right)$ for any $\left(x_{0}, u_{0}\right) \in R_{+}^{2}$. Applying Itô's formula to $\ln x(t)$, we have

$$
\mathrm{d} \ln x(t)=(b(t)-a(t) x(t)-c(t) u(t)) \mathrm{d} t+\sigma(t) \mathrm{d} B(t) .
$$

Then we have

$$
\ln x(t)=\ln x_{0}+\int_{0}^{t}(b(s)-a(s) x(s)-c(s) u(s)) \mathrm{d} s+M(t),
$$

where $M(t)=\int_{0}^{t} \sigma(s) \mathrm{d} B(s)$. By the second equation of system (1.3) we have

$$
u(t)-u_{0}=-\int_{0}^{t} e(s) u(s) \mathrm{d} s+\int_{0}^{t} f(s) x(s) \mathrm{d} s
$$

Note that $M(t)$ is a local martingale. Making use of the strong law of large numbers for local martingales (see Mao [27]), we have

$$
\lim _{t \rightarrow \infty} \frac{M(t)}{t}=0 \quad \text { a.s. }
$$

We denote $\Omega_{0}=\left\{\lim _{t \rightarrow \infty} M(t) / t=0\right\}$, obviously, $\mathbb{P}\left(\Omega_{0}\right)=1$.

Theorem 4.1 If $\left(\mathrm{H}_{2}\right)$ holds and $\langle b\rangle^{*}<0$, then system (1.3) will go to extinction almost surely.

Proof For any $\omega \in \Omega_{0}$, from (4.2) we have

$$
\frac{\ln x(t, \omega)}{t} \leq \frac{\ln x_{0}}{t}+\frac{1}{t} \int_{0}^{t} b(s) \mathrm{d} s+\frac{M(t, \omega)}{t} .
$$

Making use of (4.4) we obtain

$$
\limsup _{t \rightarrow \infty} \frac{\ln x(t, \omega)}{t} \leq\langle b\rangle^{*}
$$

That is to say, $\lim _{t \rightarrow \infty} x(t, \omega)=0$ for $\langle b\rangle^{*}<0$. Now, we will prove $\lim _{t \rightarrow \infty} u(t, \omega)=0$. Since $\lim _{t \rightarrow \infty} x(t, \omega)=0$, then for any $\alpha_{0}>0$, there is a positive constant $T_{0}$ such that

$$
|x(t, \omega)|<\frac{\alpha_{0}}{f_{u}} \quad \text { for all } t \geq T_{0} .
$$


Consequently, from (4.3) we have

$$
\frac{\mathrm{d} u(t, \omega)}{\mathrm{d} t} \leq-e(t) u(t, \omega)+\alpha_{0} \quad \text { for all } t \geq T_{0} .
$$

We consider the comparison equation

$$
\frac{\mathrm{d} v(t)}{\mathrm{d} t}=-e(t) v(t)+\alpha_{0} .
$$

By $\left(\mathrm{H}_{2}\right)$ and Lemma 2.3 with $m(t) \equiv 0$ and $v(0)=0$, we see for any positive constant $\varepsilon$ that there are constants $\delta=\delta(\varepsilon)$ and $T_{1}=T_{1}\left(u\left(T_{0}\right)\right)>T_{0}$ such that when $\left|\alpha_{0}\right|<\delta$, we have

$$
|v(t)|<\varepsilon \quad \text { for all } t \geq T_{1},
$$

where $v(t)$ is the solution of system (4.6) with initial condition $v\left(T_{0}\right)=u\left(T_{0}, \omega\right)$. Therefore, by the comparison theorem, we obtain

$$
u(t, \omega)<\varepsilon \quad \text { for all } t \geq T_{1} .
$$

Since $\varepsilon$ is arbitrary, we have $\lim _{t \rightarrow \infty} u(t, \omega)=0$. This complete the proof of the theorem, for $\mathbb{P}\left(\Omega_{0}\right)=1$.

Remark 4.1 If $c(t) \equiv 0$, we can obtain system (3.8). In Theorem 7 in [22], the authors obtained the extinction of system (3.8) under the same conditions with Theorem 4.1. Hence, if $\langle b\rangle^{*}<0$, the feedback control cannot change the extinction of the species $x$.

Theorem 4.2 Suppose $\langle b\rangle^{*}=0$, we have

(i) if $\langle a\rangle_{*}>0,\langle c\rangle_{*}>0$, and $\left(\mathrm{H}_{2}\right)$ hold, then $\liminf _{t \rightarrow \infty} x(t)=0$ and $\liminf _{t \rightarrow \infty} u(t)=0$ a.s.;

(ii) if $a_{l}, e_{l}>0$, then system (1.3) will be non-persistent in the mean a.s.

Proof (i) First of all, we will prove $\liminf _{t \rightarrow \infty} x(t, \omega)=0$ for all $\omega \in \Omega_{0}$. Otherwise, there is a positive constant $\varepsilon_{0}$ such that

$$
\liminf _{t \rightarrow \infty} x\left(t, \omega_{0}\right)>\varepsilon_{0} \quad \text { for some } \omega_{0} \in \Omega_{0} .
$$

Hence, by $\langle a\rangle_{*}>0$ and $\langle b\rangle^{*}=0$, for any positive constant $\varepsilon<\varepsilon_{0}$ there is a positive constant $T_{0}$ such that

$$
x\left(t, \omega_{0}\right) \geq \varepsilon \quad \text { and } \quad \frac{\left|M\left(t, \omega_{0}\right)\right|}{t}<\frac{\langle a\rangle_{*}}{8} \varepsilon \quad \text { for all } t \geq T_{0} .
$$

And there is a positive constant $T_{1}=T_{1}(\varepsilon)>T_{0}$ such that

$$
\frac{1}{t} \int_{T_{0}}^{t}(b(s)-\varepsilon a(s)) \mathrm{d} s<-\frac{\langle a\rangle_{*}}{2} \varepsilon \quad \text { for all } t \geq T_{1} .
$$

Then from (4.2), (4.7), and (4.8) we have

$$
\begin{aligned}
\ln x\left(t, \omega_{0}\right)-\ln x\left(T_{0}, \omega_{0}\right) & \leq \int_{T_{0}}^{t}(b(s)-a(s) \varepsilon) \mathrm{d} s+M\left(t, \omega_{0}\right)-M\left(T_{0}, \omega_{0}\right) \\
& \leq-\frac{\langle a\rangle_{*}}{4} t \quad \text { for all } t \geq T_{1} .
\end{aligned}
$$


Consequently, we have

$$
x\left(t, \omega_{0}\right) \leq x\left(T_{0}, \omega_{0}\right) \exp \left\{-\frac{\langle a\rangle_{*}}{4} t\right\} .
$$

Letting $t \rightarrow \infty$ we have $\lim \sup _{t \rightarrow \infty} x\left(t, \omega_{0}\right) \leq 0$, which is a contradiction. Therefore,

$$
\liminf _{t \rightarrow \infty} x(t, \omega)=0 \quad \text { for all } \omega \in \Omega_{0}
$$

Now, we will prove $\liminf _{t \rightarrow \infty} u(t, \omega)=0$ for all $\omega \in \Omega_{0}$. Otherwise, there is a $\eta_{0}>0$ such that

$$
\liminf _{t \rightarrow \infty} u\left(t, \omega_{0}\right) \geq 2 \eta_{0} \quad \text { for some } \omega_{0} \in \Omega_{0} .
$$

Consequently, we see that there is a positive constant $T_{0}$ such that

$$
u\left(t, \omega_{0}\right) \geq \eta_{0} \quad \text { for all } t \geq T_{0} .
$$

From (4.2) we can obtain

$$
\ln x\left(t, \omega_{0}\right)-\ln x\left(T_{0}, \omega_{0}\right) \leq \int_{T_{0}}^{t}\left(b(s)-\eta_{0} c(s)\right) \mathrm{d} s+M\left(t, \omega_{0}\right)-M\left(T_{0}, \omega_{0}\right)
$$

for all $t \geq T_{0}$. Dividing the two side of above equation by $t$ and letting $t \rightarrow \infty$, we can get

$$
\limsup _{t \rightarrow \infty} \frac{\ln x\left(t, \omega_{0}\right)}{t} \leq\langle b\rangle^{*}-\eta_{0}\langle c\rangle_{*}<0 \text { for }\langle c\rangle_{*}>0
$$

This leads to $\lim _{t \rightarrow \infty} x\left(t, \omega_{0}\right)=0$. By the proof of Theorem 4.1 we can obtain $\lim _{t \rightarrow \infty} u(t$, $\left.\omega_{0}\right)=0$. This is a contradiction. Therefore, the proof of (i) is completed.

(ii) $\langle b\rangle^{*}=0$ and (4.4) imply that, for any $\varepsilon>0$ and $\omega \in \Omega_{0}$, there is a positive constant $T_{0}$ such that

$$
\int_{0}^{t} b(s) \mathrm{d} s \leq \frac{\varepsilon t}{2} \quad \text { and } \quad M(t, \omega) \leq \frac{\varepsilon t}{2} \quad \text { for all } t \geq T_{0} .
$$

Then it follows from (4.2) that

$$
\ln x(t, \omega) \leq \ln x_{0}+\varepsilon t-a_{l} \int_{0}^{t} x(s, \omega) \mathrm{d} s
$$

Let $h(t)=\int_{0}^{t} x(s, \omega) \mathrm{d} s$, then we deduce that

$$
e^{a_{l} h(t)} \mathrm{d} h(t) \leq x_{0} e^{\varepsilon t} \mathrm{~d} t
$$

Integrating this inequality from $T>T_{0}$ to $t$ results in

$$
e^{a_{l} h(t)} \leq e^{a_{l} h(T)}+\frac{x_{0} a_{l}}{\varepsilon}\left(e^{\varepsilon t}-e^{\varepsilon T}\right) .
$$


It follows that

$$
h(t) \leq \frac{1}{a_{l}} \ln \left(e^{a_{l} h(T)}+\frac{x_{0} a_{l}}{\varepsilon}\left(e^{\varepsilon t}-e^{\varepsilon T}\right)\right) .
$$

Using L'Hospital's rule we get

$$
\limsup _{t \rightarrow \infty} \frac{h(t)}{t} \leq \frac{\varepsilon}{a_{l}}
$$

Since $\varepsilon$ is arbitrary and $x(t, \omega)>0(t>0)$, we can obtain $\lim _{t \rightarrow \infty}\langle x(t, \omega)\rangle=0$.

Now, we will prove $\lim _{t \rightarrow \infty}\langle u(t, \omega)\rangle=0$. Dividing both sides of equation (4.3) by $t$, we get

$$
\begin{aligned}
\frac{1}{t} \int_{0}^{t} u(s, \omega) \mathrm{d} s & \leq \frac{f_{u}}{e_{l} t} \int_{0}^{t} x(s, \omega) \mathrm{d} s-\frac{u(t, \omega)}{e_{l} t}+\frac{u_{0}}{e_{l} t} \\
& \leq \frac{f_{u}}{e_{l} t} \int_{0}^{t} x(s, \omega) \mathrm{d} s+\frac{u_{0}}{e_{l} t} .
\end{aligned}
$$

From $\lim _{t \rightarrow \infty}\langle x(t, \omega)\rangle=0$, letting $t \rightarrow \infty$ we obtain $\lim _{t \rightarrow \infty}\langle u(t, \omega)\rangle=0$. Since $\mathbb{P}\left(\Omega_{0}\right)=1$, this completes the proof of the theorem.

Theorem 4.3 If $e_{l}>0$ and $\langle b\rangle^{*}>0$, then species $x$ will be weakly persistent in the mean a.s., i.e. $\langle x\rangle^{*}>0$ a.s.

Proof We claim that $\Omega_{0} \subset\left\{\langle x\rangle^{*}>0\right\}$. If the claim is not true, then $\left\{\langle x\rangle^{*}=0\right\} \cap \Omega_{0} \neq \varnothing$. By the proof of (ii) in Theorem 4.2, if $e_{l}>0$, we have $\langle u(t, \omega)\rangle^{*}=0$ for any $\omega \in\left\{\langle x\rangle^{*}=0\right\} \cap \Omega_{0}$. It is easy to see that

$$
\left\langle\left. a(t) x(t, \omega)\right|^{*} \leq a_{u}\langle x(t, \omega)\rangle^{*}=0 \quad \text { and } \quad\langle c(t) u(t, \omega)\rangle^{*} \leq c_{u}\langle u(t, \omega)\rangle^{*}=0\right.
$$

From (4.2) we get

$$
\begin{aligned}
\frac{\ln x(t, \omega)}{t}= & \frac{\ln x_{0}}{t}+\frac{1}{t} \int_{0}^{t} b(s) \mathrm{d} s-\frac{1}{t} \int_{0}^{t} a(s) x(s, \omega) \mathrm{d} s \\
& -\frac{1}{t} \int_{0}^{t} c(s) u(s, \omega) \mathrm{d} s+\frac{M(t, \omega)}{t} .
\end{aligned}
$$

Combining this equation with (4.4) and (4.9) we have

$$
\limsup _{t \rightarrow \infty} \frac{\ln x(t, \omega)}{t}=\langle b\rangle^{*}
$$

Hence, there are a positive constant $T_{0}$ and a time sequence $\left\{t_{n}\right\}$ with $t_{n} \geq T_{0}$ and $t_{n+1}-t_{n} \geq$ 1 for all $n \geq 1$ such that

$$
\frac{\ln x\left(t_{n}, \omega\right)}{t_{n}}>\frac{\langle b\rangle^{*}}{2} \text { and } \quad \frac{|M(t, \omega)|}{t}<\frac{\langle b\rangle^{*}}{8} \text { for all } t>T_{0} .
$$


Let $\bar{b}=\sup _{t \geq 0}\{|b(s)|\}$. For any positive constant $\Delta t<\min \left\{1,\langle b\rangle^{*} t_{1} /(8 \bar{b})\right\}$ from (4.2) we have

$$
\begin{aligned}
\ln x\left(t_{n}, \omega\right)-\ln x(t, \omega) & \leq \int_{t}^{t_{n}} b(s) \mathrm{d} s+M\left(t_{n}, \omega\right)-M(t, \omega) \\
& \leq \bar{b} \Delta t+\frac{\langle b\rangle^{*}}{4} t_{n} \quad \text { for all } t \in\left[t_{n}-\Delta t, t_{n}\right] .
\end{aligned}
$$

Combining with (4.10) we obtain

$$
\begin{aligned}
\ln x(t, \omega) & \geq \ln x\left(t_{n}, \omega\right)-\bar{b} \Delta t-\frac{\langle b\rangle^{*}}{4} t_{n} \\
& \geq \frac{\langle b\rangle^{*}}{4} t_{n}-\bar{b} \Delta t \\
& \geq \frac{\langle b\rangle^{*}}{8} t_{n} \quad \text { for all } t \in\left[t_{n}-\Delta t, t_{n}\right] \text { and } n \geq 1 .
\end{aligned}
$$

Consequently,

$$
\begin{aligned}
\frac{1}{t_{n}} \int_{t_{1}}^{t_{n}} x(s, \omega) \mathrm{d} s & \geq \frac{1}{t_{n}} \sum_{t_{1}<t_{m} \leq t_{n}} \int_{t_{m}-\Delta t}^{t_{m}} x(s, \omega) \mathrm{d} s \\
& \geq \frac{1}{t_{n}} \sum_{t_{1}<t_{m} \leq t_{n}} \Delta t \exp \left\{\frac{\langle b\rangle^{*}}{8} t_{m}\right\} \\
& \geq \frac{\Delta t}{t_{n}} \exp \left\{\frac{\langle b\rangle^{*}}{8} t_{n}\right\} .
\end{aligned}
$$

Since $\langle b\rangle^{*}>0, \lim _{n \rightarrow \infty} \frac{1}{t_{n}} \int_{t_{1}}^{t_{n}} x(s, \omega) \mathrm{d} s=+\infty$, which contradicts with $\omega \in\left\{\langle x\rangle^{*}=0\right\} \cap \Omega_{0}$. Therefore, $\Omega_{0} \subset\left\{\langle x\rangle^{*}>0\right\}$, i.e. $\langle x\rangle^{*}>0$ a.s.

Remark 4.2 In Theorem 9 in [22], the authors studied the weakly persistent in the mean of system (3.8) with the conditions $a_{l}>0$ and $\langle b\rangle^{*}>0$. Obviously, from Theorem 4.3 we can obtain the same result with [22] only under the condition $\langle b\rangle^{*}>0$. Therefore, the result in [22] is improved by Theorem 4.3.

Remark 4.3 In this theorem, due to shortage of the analysis techniques on the stochastic model, the weakly persistent in the mean of $u$ case has not been studied. But we can see that the feedback control does not affect the persistence property of the species $x$ under the conditions in this theorem.

Theorem 4.4 Assume $a_{l}>0, e_{l}>0, f_{l}>0$, and $\langle b\rangle_{*}>0$. Then system (1.3) will be uniform permanent in the mean a.s. Moreover,

$$
\underline{x} \leq\langle x\rangle_{*} \leq\langle x\rangle^{*} \leq \bar{x} \quad \text { and } \quad \underline{u} \leq\langle u\rangle_{*} \leq\langle u\rangle^{*} \leq \bar{u} \quad \text { a.s. }
$$

where $\underline{x}=\langle b\rangle_{*} e_{l} /\left(a_{u} e_{l}+c_{u} f_{u}\right), \underline{u}=f_{l} e_{l}\langle b\rangle_{*} / e_{u}\left(a_{u} e_{l}+c_{u} f_{u}\right), \bar{x}=\left(\langle b\rangle^{*} e_{u}\left(a_{u} e_{l}+c_{u} f_{u}\right)-c_{l} f_{l} e_{l}\langle b\rangle_{*}\right) /$ $a_{l} e_{u}\left(a_{u} e_{l}+c_{u} f_{u}\right)$, and $\bar{u}=f_{u}\left(\langle b\rangle^{*} e_{u}\left(a_{u} e_{l}+c_{u} f_{u}\right)-c_{l} f_{l} e_{l}\langle b\rangle_{*}\right) / a_{l} e_{l} e_{u}\left(a_{u} e_{l}+c_{u} f_{u}\right)$.

Proof From equation (4.3) we have

$$
\int_{0}^{t} e(s) u(s) \mathrm{d} s=\int_{0}^{t} f(s) x(s) \mathrm{d} s-u(t)+u_{0} \leq f_{u} \int_{0}^{t} x(s) \mathrm{d} s+u_{0} .
$$


Consequently, we have

$$
\int_{0}^{t} u(s) \mathrm{d} s \leq \frac{f_{u}}{e_{l}} \int_{0}^{t} x(s) \mathrm{d} s+\frac{u_{0}}{e_{l}} .
$$

For any $\varepsilon>0$ and $\omega \in \Omega_{0}$, there is a $T$ such that

$$
\langle b(t)\rangle>\langle b\rangle_{*}-\frac{\varepsilon}{2} \quad \text { and } \quad \frac{M(t)}{t}>-\frac{\varepsilon}{2} \quad \text { for all } t>T .
$$

Substituting these inequalities and (4.11) into equation (4.2) we get

$$
\ln x(t)-\ln x_{0} \geq v t-\left(a_{u}+\frac{c_{u} f_{u}}{e_{l}}\right) \int_{0}^{t} x(s) \mathrm{d} s-\frac{c_{u} u_{0}}{e_{l}} \quad \text { for all } t \geq T
$$

where $v=\langle b\rangle_{*}-\varepsilon$. Let $g(t)=\int_{0}^{t} x(s) \mathrm{d} s$, then we have

$$
\ln \frac{\mathrm{d} g(t)}{\mathrm{d} t}-\ln x_{0} \geq v t-\frac{c_{u} u_{0}}{e_{l}}-\left(a_{u}+\frac{c_{u} f_{u}}{e_{l}}\right) g(t) .
$$

Consequently,

$$
\exp \left\{\left(a_{u}+\frac{c_{u} f_{u}}{e_{l}}\right) g(t)\right\} \frac{\mathrm{d} g(t)}{\mathrm{d} t} \geq x_{0} \exp \left\{v t-\frac{c_{u} u_{0}}{e_{l}}\right\} .
$$

Integrating this inequality from $T$ to $t$ we have

$$
\exp \left\{\left(a_{u}+\frac{c_{u} f_{u}}{e_{l}}\right) g(t)\right\} \geq \exp \left\{\left(a_{u}+\frac{c_{u} f_{u}}{e_{l}}\right) g(T)\right\}+\frac{x_{0}\left(a_{u}+\frac{c_{u} f_{u}}{e_{l}}\right)}{\nu \exp \left\{\frac{c_{u} u_{0}}{e_{l}}\right\}}(\exp \{v t\}-\exp \{v T\}) .
$$

Taking the logarithm of both sides yields

$$
g(t) \geq\left(a_{u}+\frac{c_{u} f_{u}}{e_{l}}\right)^{-1} \ln \left(\exp \left\{\left(a_{u}+\frac{c_{u} f_{u}}{e_{l}}\right) g(T)\right\}+\frac{x_{0}\left(a_{u}+\frac{c_{u} f_{u}}{e_{l}}\right)}{v \exp \left\{\frac{c_{u} u_{0}}{e_{l}}\right\}}(\exp \{v t\}-\exp \{v T\})\right) .
$$

That is to say,

$$
\begin{aligned}
\langle x\rangle_{*} \geq & \liminf _{t \rightarrow \infty}\left[\left(a_{u}+\frac{c_{u} f_{u}}{e_{l}}\right) t\right]^{-1} \\
& \times \ln \left(\exp \left\{\left(a_{u}+\frac{c_{u} f_{u}}{e_{l}}\right) g(T)\right\}+\frac{x_{0}\left(a_{u}+\frac{c_{u} f_{u}}{e_{l}}\right)}{v \exp \left\{\frac{c_{u} u_{0}}{e_{l}}\right\}}(\exp \{v t\}-\exp \{v T\})\right) .
\end{aligned}
$$

Using L'Hospital's rule, we can obtain

$$
\langle x\rangle_{*} \geq \frac{v}{a_{u}+\frac{c_{u} f_{u}}{e_{l}}}=\frac{v e_{l}}{a_{u} e_{l}+c_{u} f_{u}} .
$$

Since $\varepsilon$ is arbitrary, we obtain

$$
\langle x\rangle_{*} \geq \frac{\langle b\rangle_{*} e_{l}}{a_{u} e_{l}+c_{u} f_{u}}:=\underline{x} \quad \text { for all } \omega \in \Omega_{0} .
$$


Now, we will prove $\langle u\rangle_{*}$ also has a lower bound. From the above proof, we can see for any $\varepsilon>0$ and $\omega \in \Omega_{0}$ that there is a positive constant $T$ such that

$$
\int_{0}^{t} x(s) \mathrm{d} s \geq(\underline{x}-\varepsilon) t \quad \text { for all } t \geq T .
$$

Substituting the above inequality into (4.3), we have

$$
u(t) \geq-e_{u} \int_{0}^{t} u(s) \mathrm{d} s+f_{l}(\underline{x}-\varepsilon) t \quad \text { for all } t \geq T .
$$

Let $h(t)=\int_{0}^{t} u(s) \mathrm{d} s$, then we have

$$
\frac{\mathrm{d} h(t)}{\mathrm{d} t} \geq-e_{u} h(t)+f_{l}(\underline{x}-\varepsilon) t \quad \text { for all } t \geq T .
$$

Consider the following comparison equation:

$$
\frac{\mathrm{d} y(t)}{\mathrm{d} t}=-e_{u} y(t)+f_{l}(\underline{x}-\varepsilon) t
$$

with initial value $y(T)=h(T)$. By the well-known variation-of-constants formula, we have

$$
\begin{aligned}
y(t) & =f_{l}(\underline{x}-\varepsilon) \int_{T}^{t} s \exp \left\{-e_{u}(t-s)\right\} \mathrm{d} s+h(T) \exp \left\{-e_{u}(t-T)\right\} \\
& =\frac{f_{l}}{e_{u}}(\underline{x}-\varepsilon)\left(\left(t-\frac{1}{e_{u}}\right)-\left(T-\frac{1}{e_{u}}\right) \exp \left\{-e_{u}(t-T)\right\}\right)+h(T) \exp \left\{-e_{u}(t-T)\right\} .
\end{aligned}
$$

By the comparison theorem, we have

$$
\liminf _{t \rightarrow \infty} \frac{h(t)}{t} \geq \liminf _{t \rightarrow \infty} \frac{y(t)}{t}=\frac{f_{l}}{e_{u}}(\underline{x}-\varepsilon) .
$$

Since $\varepsilon$ is arbitrary, we obtain

$$
\langle u\rangle_{*}=\liminf _{t \rightarrow \infty} \frac{h(t)}{t} \geq \frac{f_{l}}{e_{u}} \underline{x}:=\underline{u} .
$$

In the following, we will prove the upper bound of $\langle x\rangle^{*}$ and $\langle u\rangle^{*}$. From (4.4) and (4.12), for any $\varepsilon>0$ and $\omega \in \Omega_{0}$ there exists a positive constant $T_{0}$ such that

$$
\int_{0}^{t} b(s) \mathrm{d} s \leq\left(\langle b\rangle^{*}+\frac{c_{l} \varepsilon}{3}\right) t, \quad \int_{0}^{t} u(s) \mathrm{d} s \geq\left(\underline{u}-\frac{\varepsilon}{3}\right) t \quad \text { and } \quad M(t) \leq \frac{c_{l} \varepsilon}{3} t
$$

for all $t \geq T_{0}$. Substituting (4.13) into equation (4.2) we have

$$
\begin{aligned}
\ln x(t)-\ln x_{0} & \leq\left(\langle b\rangle^{*}+\frac{c_{l} \varepsilon}{3}\right) t-a_{l} \int_{0}^{t} x(s) \mathrm{d} s-c_{l}\left(\underline{u}-\frac{\varepsilon}{3}\right) t+\frac{c_{l} \varepsilon}{3} t \\
& =\left(\langle b\rangle^{*}-c_{l}(\underline{u}-\varepsilon)\right) t-a_{l} \int_{0}^{t} x(s) \mathrm{d} s \quad \text { for all } t \geq T_{0} .
\end{aligned}
$$


Let $k(t)=\int_{0}^{t} x(s) \mathrm{d} s$, then we have

$$
\ln \frac{\mathrm{d} k(t)}{\mathrm{d} t}-\ln x_{0} \leq \rho t-a_{l} k(t) \quad \text { for all } t \geq T_{0}
$$

where $\rho=\langle b\rangle^{*}-c_{l}(\underline{u}-\varepsilon)$. Consequently,

$$
\exp \left\{a_{l} k(t)\right\} \frac{\mathrm{d} k(t)}{\mathrm{d} t} \leq x_{0} \exp \{\rho t\} \quad \text { for all } t \geq T_{0} .
$$

Integrating this inequality from $T_{0}$ to $t$ we have

$$
\exp \left\{a_{l} k(t)\right\} \leq \exp \left\{a_{l} k\left(T_{0}\right)\right\}+\frac{a_{l} x_{0}}{\rho}\left(\exp \{\rho t\}-\exp \left\{\rho T_{0}\right\}\right) .
$$

Taking the logarithm of both sides yields

$$
k(t) \leq \frac{1}{a_{l}} \ln \left\{\exp \left\{a_{l} k\left(T_{0}\right)\right\}+\frac{a_{l} x_{0}}{\rho}\left(\exp \{\rho t\}-\exp \left\{\rho T_{0}\right\}\right)\right\} .
$$

That is to say,

$$
\langle x\rangle^{*} \leq \limsup _{t \rightarrow \infty} \frac{1}{a_{l} t} \ln \left\{\exp \left\{a_{l} k\left(T_{0}\right)\right\}+\frac{a_{l} x_{0}}{\rho}\left(\exp \{\rho t\}-\exp \left\{\rho T_{0}\right\}\right)\right\} .
$$

Using L'Hospital's rule, we can obtain $\langle x\rangle^{*} \leq \rho / a_{l}$. Since $\varepsilon$ is arbitrary, we obtain

$$
\langle x\rangle^{*} \leq \frac{\langle b\rangle^{*}-c_{l} \underline{u}}{a_{l}}:=\bar{x} .
$$

Rewriting equation (4.3) we have

$$
\begin{aligned}
\int_{0}^{t} u(s) \mathrm{d} s & \leq \frac{f_{u}}{e_{l}} \int_{0}^{t} x(s) \mathrm{d} s-\frac{u(t)}{e_{l}}+\frac{u_{0}}{e_{l}} \\
& \leq \frac{f_{u}}{e_{l}} \int_{0}^{t} x(s) \mathrm{d} s+\frac{u_{0}}{e_{l}} .
\end{aligned}
$$

Combining this inequality with equation (4.14), we have $\langle u\rangle^{*} \leq f_{u} \bar{x} / e_{l}:=\bar{u}$. This completes the proof.

Remark 4.4 From Theorems 4.1-4.4, we can find that the feedback control is harmless to the permanence of the species under the randomized environment.

\section{Numerical simulation}

In this section we use the Milstein method mentioned in Higham [29] to substantiate the analytical findings. For system (1.3), consider the discretization equation:

$$
\left\{\begin{aligned}
x_{k+1}= & x_{k}+x_{k}\left[r(k \Delta t)-a(k \Delta t) x_{k}-c(k \Delta t) u_{k}\right] \Delta t+\sigma(k \Delta t) x_{k} \sqrt{\Delta t} \xi_{k} \\
& +0.5 \sigma^{2}(k \Delta t) x_{k}\left(\xi_{k}^{2} \Delta t-\Delta t\right), \\
u_{k+1}= & u_{k}-e(k \Delta t) u_{k}+f(k \Delta t) x_{k},
\end{aligned}\right.
$$

where $\xi_{k}$ is a Gaussian random variable that follows $N(0,1)$. 
Figure 1 Extinction.

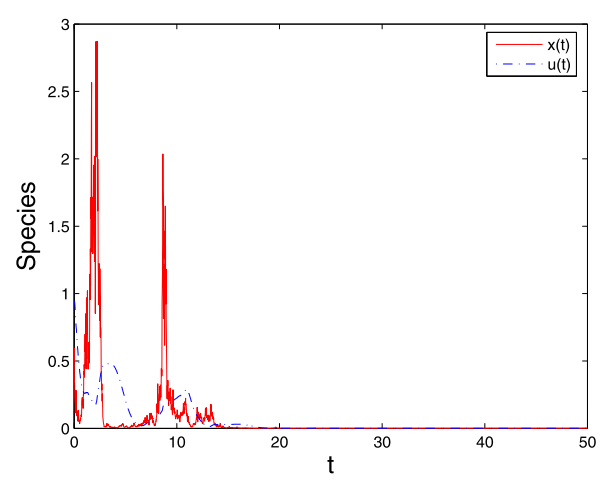

Figure 2 Weakly persistent in the mean.
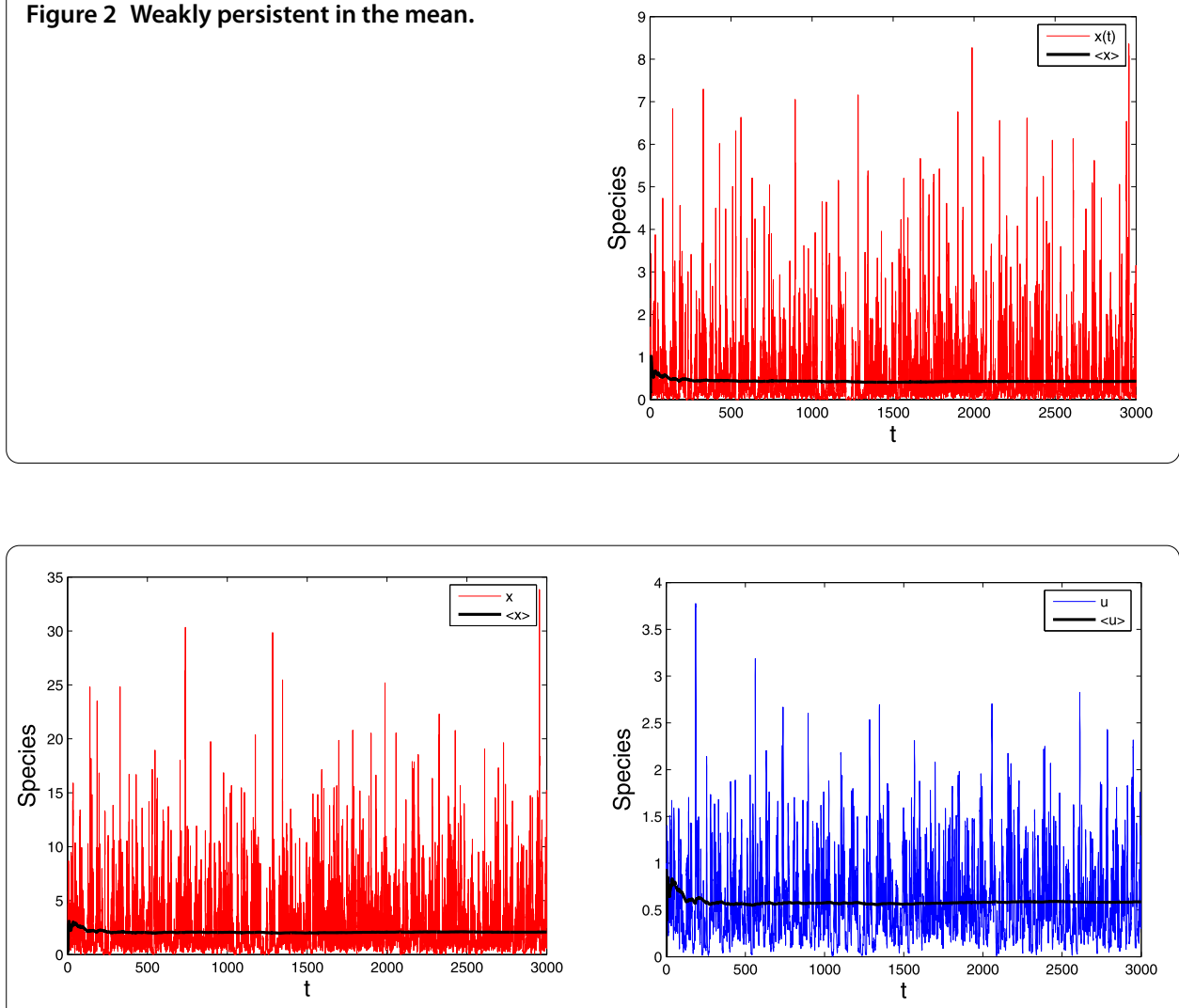

Figure 3 Persistent in the mean of $x$ (the left figure) and persistent in the mean of $u$ (the right figure).

In Figure 1, we choose $r(t)=1+\sin t, a(t)=0.2+0.2 \sin t, \sigma^{2}(t) / 2=1.5+0.5 \sin t, c(t)=$ $1+\sin t, e(t)=1+\cos t$, and $f(t)=1+\cos \sqrt{3} t$. Then it is easy to obtain $\langle b\rangle^{*}=-0.5<0$ and $\int_{t}^{t+2 \pi} e(s) \mathrm{d} s=1>0$. In view of Theorem 4.1, $x$ and $u$ will go to extinction. Figure 1 confirms this.

In Figure 2, we choose $r(t)=1+\sin t, a(t)=0.2+0.2 \sin t, \sigma^{2}(t) / 2=0.5+0.5 \sin t, c(t)=$ $1+\sin t, e(t)=1+0.5 \cos t$, and $f(t)=1+\cos \sqrt{3} t$. Then the conditions $\langle b\rangle^{*}=0.5>0$ and $e_{l}=0.5>0$ are valid. By virtue of Theorem 4.3, $x$ will be weakly persistent in the mean. This can be seen from Figure 2 . 
In Figure 3, we choose $r(t)=1+\sin t, a(t)=0.2+0.1 \sin t, \sigma^{2}(t) / 2=0.5+0.5 \sin t$, $c(t)=0.15+0.05 \sin t, e(t)=0.75+0.25 \cos t$, and $f(t)=0.2+0.1 \cos \sqrt{3} t$. Then it is easy to obtain $\langle b\rangle^{*}=0.5>0, a_{l}=0.1, a_{u}=0.3, c_{l}=0.1, c_{u}=0.2, e_{l}=0.5, e_{u}=1, f_{l}=0.1, f_{u}=0.3$. Consequently, we have $\underline{x}=1.1904, \bar{x}=4.8810, \underline{u}=0.1190$, and $\bar{u}=2.9286$. Applying Theorem 4.4, $x$ and $u$ will be persistent in the mean. Figure 3(a) and (b) confirms this.

\section{Future directions}

Recently, some scholars studied some interesting problems, such as model with jumps (see $[30,31])$ and model with time delay (see [32, 33]). It is an interesting question to investigate the dynamics property of the stochastic species systems with feedback control, jumps, and time delay. This will be our future work.

\section{Competing interests}

The authors declare that they have no competing interests.

\section{Authors' contributions}

All authors completed the paper together. All authors read and approved the final manuscript.

\section{Author details}

${ }^{1}$ College of Science, University of Shanghai for Science and Technology, Shanghai, 200093, P.R. China. ${ }^{2}$ School of Science, Anhui Agriculture University, Hefei, 230036, P.R. China.

\section{Acknowledgements}

We thank the National Natural Science Foundation of China (grant number: 11401382) and Hujiang Foundation of China (grant number: B14005).

Received: 24 October 2015 Accepted: 29 February 2016 Published online: 18 July 2016

\section{References}

1. May, RM: Stability and Complexity in Model Ecosystems. Princeton University Press, Princeton (1973)

2. Freedman, HI, Wu, J: Periodic solutions of single-species models with periodic delay. SIAM J. Math. Anal. 23, 689-701 (1992)

3. Golpalsamy, K: Stability and Oscillations in Delay Differential Equations of Population Dynamics. Kluwer Academic, Dordrecht (1992)

4. Kuang, Y: Delay Differential Equations with Applications in Population Dynamics. Academic Press, Boston (1993)

5. Lisena, B: Global attractivity in nonautonomous logistic equations with delay. Nonlinear Anal., Real World Appl. 9, 53-63 (2008)

6. Golpalsamy, K, Weng, P: Feedback regulation of logistic growth. Int. J. Math. Sci. 16(1), 177-192 (1993)

7. Chen, FD: Global stability of a single species model with feedback control and distributed time delay. Appl. Math. Comput. 178, 474-479 (2006)

8. Yang, F, Jiang, D: Existence and global attractivity of positive periodic solution of a logistic growth system with feedback control and deviating arguments. Ann. Differ. Equ. 17, 377-384 (2001)

9. Hou, H, Li, W: Positive periodic solutions of a class of delay differential system with feedback control. Appl. Math. Comput. 148, 35-46 (2004)

10. Chen, $F, C h e n, X, C h a o, J:$ Positive periodic solutions of a class of non-autonomous single species population model with delays and feedback control. Acta Math. Sin. 21, 1319-1339 (2005)

11. $\mathrm{Xu}, \mathrm{J}$, Teng, Z: Permanence for a nonautonomous discrete single-species system with delays and feedback control. Appl. Math. Lett. 23, 949-954 (2010)

12. $\mathrm{Hu}, \mathrm{H}, \mathrm{Teng}, \mathrm{Z}$, Jiang, $\mathrm{H}$ : On the permanence in non-autonomous Lotka-Volterra competitive system with pure-delays and feedback controls. Nonlinear Anal., Real World Appl. 10, 1803-1815 (2009)

13. $\mathrm{Hu}, \mathrm{H}, \mathrm{Teng}, \mathrm{Z}, \mathrm{Gao}, \mathrm{S}$ : Extinction in nonautonomous Lotka-Volterra competitive system with pure-delays and feedback controls. Nonlinear Anal., Real World Appl. 10, 2508-2520 (2009)

14. Gard, TC: Persistence in stochastic food web models. Bull. Math. Biol. 46, 357-370 (1984)

15. Gard, TC: Stability for multispecies population models in random environments. Nonlinear Anal. 10, 1411-1419 (1986)

16. Mao, X, Marion, G, Renshaw, E: Environmental Brownian noise suppresses explosions in population dynamics. Stoch. Process. Appl. 97, 95-110 (2002)

17. Bahar, A, Mao, X: Stochastic delay Lotka-Volterra model. J. Math. Anal. Appl. 292, 364-380 (2004)

18. Bahar, A, Mao, X: Stochastic delay population dynamics. Int. J. Pure Appl. Math. 11, 377-400 (2004)

19. Jiang, D, Shi, N, Zhao, Y: Existence, uniqueness, and global stability of positive solutions to the food-limited population model with random perturbation. Math. Comput. Model. 42, 651-658 (2005)

20. Mao, X: Delay population dynamics and environmental noise. Stoch. Dyn. 5, 149-162 (2005)

21. Mao, X, Marion, G, Renshaw, E: Asymptotic behavior of the stochastic Lotka-Volterra model. J. Math. Anal. Appl. 287, 141-156 (2003)

22. Liu, M, Wang, K: Persistence and extinction in stochastic non-autonomous logistic systems. J. Math. Anal. Appl. 375 , 443-457 (2011) 
23. Jiang, DQ, Shi, NZ, Li, XY: Global stability and stochastic permanence of a non-autonomous logistic equation with random perturbation. J. Math. Anal. Appl. 340, 588-597 (2008)

24. Jiang, DQ, Shi, NZ: A note on non-autonomous logistic equation with random perturbation. J. Math. Anal. Appl. 303, 164-172(2005)

25. Teng, ZD, Li, ZM: Permanence and asymptotic behavior of the $n$-species nonautonomous Lotka-Volterra competitive systems. Comput. Math. Appl. 39, 107-116 (2000)

26. $\mathrm{Hu}, \mathrm{H}$, Teng, Z, Jiang, $\mathrm{H}$ : Permanence of the nonautonomous competitive systems with infinite delay and feedback controls. Nonlinear Anal., Real World Appl. 10, 2420-2433 (2009)

27. Mao, X: Stochastic Differential Equations and Applications. Horwood, Chichester (1997)

28. Ikeda, N: Stochastic Differential Equations and Diffusion Processes. North-Holland, Amsterdam (1981)

29. Higham, DJ: An algorithmic introduction to numerical simulation of stochastic differential equations. SIAM Rev. 43, 525-546 (2001)

30. Liu, M, Wang, K: Stochastic Lotka-Volterra systems with Levy noise. J. Math. Anal. Appl. 410, 750-763 (2014)

31. Bao, J, Mao, X, Yin, G, Yuan, C: Competitive Lotka-Volterra population dynamics with jumps. Nonlinear Anal. 74, 6601-6616 (2011)

32. Liu, M, Bai, C: Optimal harvesting of a stochastic logistic model with time delay. J. Nonlinear Sci. 25, 277-289 (2015)

33. Liu, M, Bai, C: On a stochastic delayed predator-prey model with Levy jumps. Appl. Math. Comput. 228, 563-570 (2014)

\section{Submit your manuscript to a SpringerOpen ${ }^{\ominus}$ journal and benefit from:}

- Convenient online submission

Rigorous peer review

- Immediate publication on acceptance

- Open access: articles freely available online

- High visibility within the field

- Retaining the copyright to your article 